\title{
The effects of interruptions in work activity: field and laboratory results
}

\author{
Hélène Eyrolle*, Jean-Marie Cellier \\ Laboratoire Travail et Cognition, UMR CNRS 5551, Maison de la Recherche, Université Toulouse Le Mirail, 5 allées A. Machado, \\ F - 31058 Toulouse cédex 1, France
}

Received 13 March 1997; accepted 9 September 1999

\begin{abstract}
The effects of interruptions in work activity were investigated, first in a field study where the operators' task was to card-index data about customers' phone lines. The interruptions due to customers' calls resulted in an increase of the processing time of the current task and in the use of several management strategies. A laboratory study was then designed in order to study the effects of temporal strain, complexity and similarity on time-sharing efficiency and to clarify the psychological mechanisms underlying the switching from one task to the other. The results showed especially a significant effect of temporal strains on performance and a strong increase in mean error rate at the very beginning of the processing of the second task. In conclusion, advice is given for both technical and organisational design. (C) 2000 Elsevier Science Ltd. All rights reserved.
\end{abstract}

Keywords: Interference; Interruptions; Time-sharing; Work activity

\section{Introduction}

Our interest to study the effects of interruptions in work activity comes from several demands (for instance from the Ministry of Defence and from a telecommunications office). The questions shared by these organisations were: why does an operator interrupt his main task? What are the consequences of these interruptions on his on-going work and on his workload?

For the Ministry of Defence, the main question was related to attention sharing between several tasks in situations such as weapon systems driving or fighter piloting. According to the situation under study, tasks are present at the same time (time-sharing tasks) or another task occurs during the execution of the task in progress (interruptive task).

The telecommunications office was mainly interested in interruptive tasks. In the literature, interruptions in work activity are considered to be a factor that increases workload (Wickens, 1984), constitutes a job stressor (Carayon, 1994) or affects psychological well-being (Roe

\footnotetext{
* Corresponding author. Tel.: + 335-61-50-41; fax: + 335-61-50-3533.

E-mail address: eyrolle@univ-tlse2.fr (H. Eyrolle).
}

et al., 1996). In process control tasks, Bainbridge (1984) showed that incidents are often related to the occurrence of a second task that captures operators' attention and disturbs the processing of the current task. In the nuclear power industry, a study (Griffon-Fouco and Ghertman, 1984) about incidents resulting in shut-down of the plant showed that in more than $15 \%$ of these incidents, operators had been distracted during the execution of their current task. Therefore, interruptions can also reduce human reliability. Reason's model (1990) about human error assumed that some errors are related to a failure in the necessary attentional monitoring and he distinguished a particular class of errors that he called "omissions following interruptions".

These multiple effects following interruptions have oriented the analysis toward the phenomenon of interference. From a theoretical point of view, the account of this phenomenon is in keeping with the general paradigm of time-sharing tasks. Three main classes of models have been proposed to explain interference.

(1) The human is considered as a single channel of limited capacity (Broadbent, 1958; Treisman, 1964; Welford, 1967). A second task having to be processed concurrently can saturate the capacity and lead to interference. According to this approach, interference 
occurs because of the bottleneck in human information processing.

(2) The human information processing system is considered as a single (Hockey and Hamilton, 1983; Kahneman, 1973; Norman and Bobrow, 1975; Navon and Gopher, 1979) or multiple (Wickens, 1984) reservoir of resources. According to these approaches, interference occurs when the resources required by each task overlap.

(3) The nature of processing - automatic vs. controlled processing (Shiffrin and Schneider, 1977) - is put forward to account for interference. If at least one of both tasks can be carried out automatically, time sharing can be efficient. Posner and Snyder (1975) assumed that when both tasks require controlled processing, two mechanisms (facilitation and inhibition) are necessary to manage the switch from one task to the other. Operation of these mechanisms can lead to interference.

Several variables are usually considered as affecting time sharing efficiency, including those of tasks difficulty (under which are often included temporal strains and work complexity) and those of task similarity - although its facilitatory or inhibitory role is not clearly stated.

Considering the two last classes of models presented, we assumed that in a time-sharing situation the processing of two tasks depends on a strategy of resource management; the lower the demands of the task, the easier will be the management. In a task-switching situation, resources required for the new task must be activated while those used in the first task must be inhibited. Failures in either of these processes will give rise to interference. This model is compatible with that developed by Hockey and Hamilton (1983) in that it puts the emphasis on the adaptive and flexible nature of mental states.

Operationally, one can study the effects of interruption at several levels. The aim of this paper is to analyse interruptions in order to show the processing disturbances they can create when switching from one task to the other. Therefore, we present first the results of an exploratory field study in which we focussed on the effects of interruptions on performance and regulation modes. Secondly, we present results of an experimental laboratory study designed to analyse the effects of several factors on time-sharing efficiency and to clarify the psychological mechanisms underlying the switching from one task to the other.

\section{The field study}

\subsection{Task}

The field study was carried out in a commercial telecommunications office. The operators' task was to cap- ture on a computer, and to keep up to date, data about telephone lines. Operators had at their disposal a telephone and a computer. Three tasks were distinguished:

- Creation task: the operator has to constitute a file for each new consumer in which information about the consumer (name, surname, address, ...) and about the telephone line are recorded;

- Regularisation task: the file is held over and will be regularised, by an operator of the office, on the order of the technical services after the line has been put into service;

- Modification task: the operator has to modify information in afile at a customer's request (for instance, installation of a second telephonic socket or modification of the customer name).

The regularisation task is carried out from written documents transmitted by the technical services. Both other tasks are executed from customers 'letters or from customers' phone calls. This last case leads to an interruption of the ongoing task. The occurrence of these interruptive tasks cannot be predicted by the operator.

For commercial reasons, the organisation gave priority to customers' phone calls: the operators had to answer phone calls as soon as they occurred. The computing system did not allow saving of data during the processing, so a task interruption led to an irretrievable loss of data.

\subsection{Method}

Data consisted of observations of ten operators' activity during $150 \mathrm{~h}$. The processing time of each first task (with or without interruptions) and the number of interruptions were collected. Errors (related to syntax, field of record or code) were recorded when they occurred on the first tasks.

We did not ask for verbalisations from operators during the activity in order to avoid interference in the processing tasks. Nevertheless, deferred comments were requested during the breaks.

\subsection{Results}

\subsubsection{Effects of interruptive tasks on the processing of the} first tasks

Results showed an increase in the mean processing times of the first tasks when they were interrupted (Fig. 1). On the basis of the mean processing time of customers' cards carried out without interruptions, the mean processing time of the first tasks increased significantly when their processing was shared into two periods, i.e. one interruption $(t=6.58, p<0.001)$ and into three periods, i.e. two interruptions $(t=5.71, p<0.001)$. 


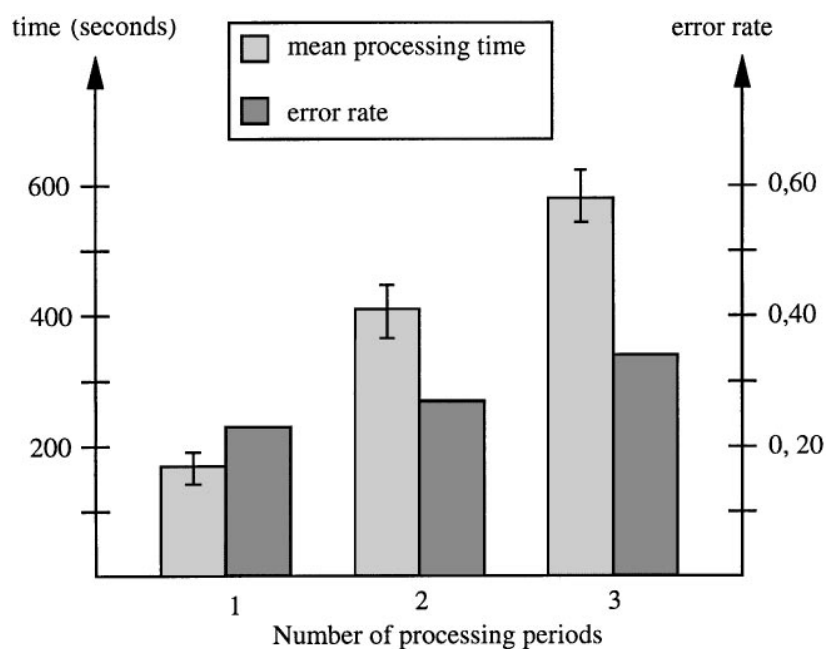

Fig. 1. Mean processing time and error rate for the first task according to the number of processing periods.

Mean error rates increased when the processing of the first tasks was shared into two or three periods, but not significantly.

These results showed that interruptions had effects on the processing of the first tasks. One can then ask whether these interruptions and their effects led operators to adopt specific regulation modes. To deal with this question we analysed the management strategies used by operators to cope with interruptive tasks.

\subsubsection{Management strategies}

Four strategies have been identified (Fig. 2) when the operator is processing the first task and a customer's call occurs.

- In strategy 1 , the processing of the first task is completed before processing the interruptive task.

- In strategy 2, the operator tells the customer something like: "please wait for a few minutes" and he completes the processing of his first task before processing the interruptive task.

- In strategy 3, the content of the interruptive task is identified, the processing of the first task is completed and then the interruptive task is processed.

- In strategy 4 , the interruptive task is immediately processed, with the processing of the first task completed later.

These strategies can be summarised as follows: the operators suspend or not the processing of the first task; they identify or not content of the interruptive task; they postpone the processing of either the first task or the interruptive task.

The most frequently used strategy was the fourth one $(77 \%)$, with strategies 1, 2 and 3 used, respectively, in 10, 7 and $6 \%$ of the cases.
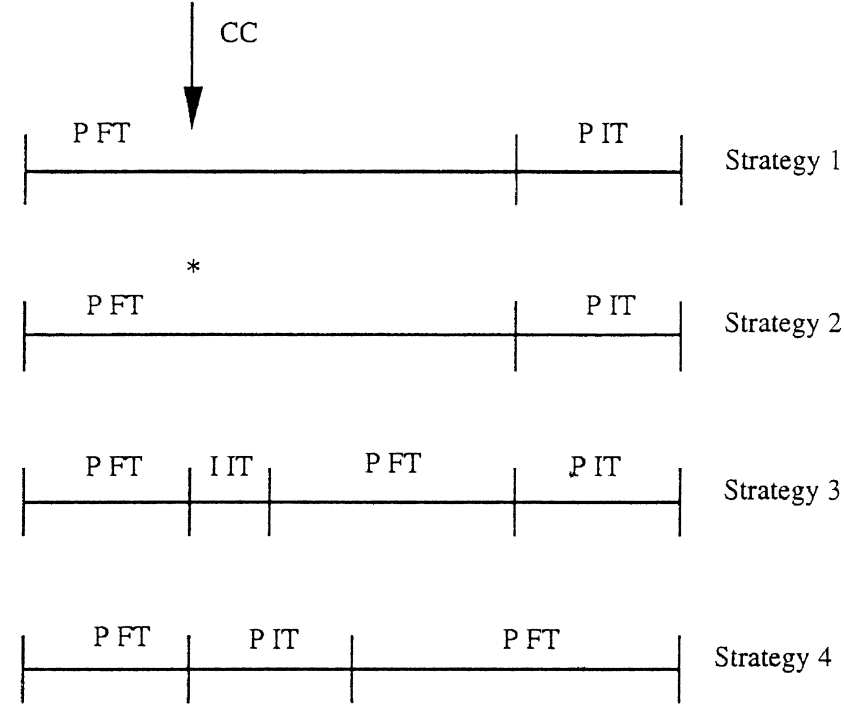

Fig. 2. Management strategies.

(CC: customers' call; PFT: processing of the first task;

PIT: processing of the interruptive task;

IIT: identification of the interruptive task;

*: the customer is asked to wait for a few minutes by the operator)

Strategies 1 and 2 are compatible with the aim to reduce the effects of the interruptions but they only account for $17 \%$ of the cases. These strategies were used after an evaluation by the operators of the time needed to complete the on-going task. If this time was short (one or two minutes), it was then less costly to complete the on-going task than to interrupt it, since the computer system did not allow them to save data before the end of the task.

Paradoxically, the most frequently used strategy (strategy 4) results in sharing the processing of the first task and therefore, on the basis of the results presented previously, produces an additional cost. This result can be accounted for by the commercial policy of the organisation that recommends immediate answers to customers' calls.

In summary, interruptions produce quantitative and qualitative effects on performance. These effects probably lead to an increased mental workload, either because of anticipations in order to avoid errors or because of effort to recover from errors. Finally, it seems that the switching from one task to the other constitutes a crucial moment that requires further in-depth investigations. These investigations are difficult to carry out in a field study and that is one of the reasons why we designed a laboratory experiment.

\section{The laboratory study}

The aim of the experimental laboratory study was to analyse the effects of several factors on time sharing 


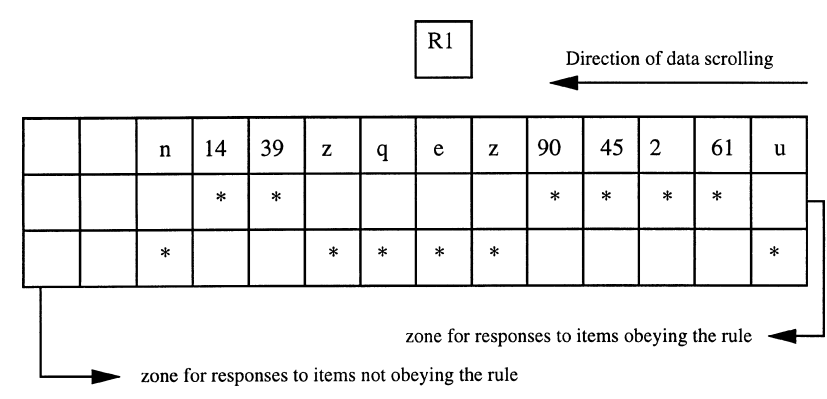

Fig. 3. A typical screen presentation.

efficiency and to clarify the psychological mechanisms underlying the switching from one task to the other.

\subsection{Tasks}

The participant was required to select particular items from a scrolling display of numerical and alphabetical information on a microcomputer. The items to be selected appeared in a window on the screen (Fig. 3). The information scrolled in a discontinuous fashion from right to left. Fourteen different pieces of information were presented. Each participant performed two of the following six tasks (rules):

- select even numbers followed by an odd number (R1),

- select even numbers followed by an even number (R2),

- select even numbers preceded by a letter and followed by a number over 50 (R3),

- select odd numbers preceded by a consonant and followed by a number over 50 (R4),

- select even numbers followed by a number over 50 (R5),

- select even numbers preceded by a vowel and followed by a number over 50 (R6).

The participant's response was made using the mouse, by moving the pointer to the row just below the selected information and clicking the button (Fig. 3). If the information did not correspond to the criterion, the participant had to make a response in the bottom row. Participants' responses were signalled by the appearance of an asterisk in the corresponding box (Fig. 3).

\subsection{Method}

The experiment took place in four stages, and each participant carried out each of the stages. Half of the participants in each group carried out stage 3 before stage 2 , the other half performed each stage in the normal sequence. This reversal did not affect the results for stage 3 , and is thus not taken into account in the presentation of the results. The stages were:

Stage 1: learning each task separately (10 min per task)

Stage 2: dual task (10 min). A second window like that shown in Fig. 3 was displayed on the screen. The subject

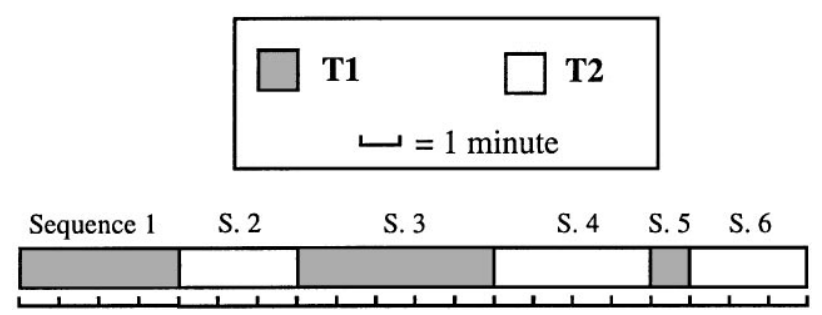

Fig. 4. Duration of each task in stage 3.

had to carry out both tasks simultaneously in their separate windows.

Stage 3: task switching (20 min). This stage was carried out in 6 sequences (Fig. 4). Each participant carried out all six sequences in the same order. Only one window was displayed on the screen. When the second task was about to be presented, the participant was warned by a tone and the appearance of the @ character on the screen above the data zone. The unpredictability in timing of the second task and the duration of presentation were varied in a pseudo-random manner within the following constraints: each task was presented 3 times to each group of participants (6 sequences for stage 3 ); for each task, the minimum duration was $1 \mathrm{~min}$ and the maximum $5 \mathrm{~min}$.

Stage 4: single-task situation ( 5 min per task)

We present here only the results obtained during stage 3 , as these are directly relevant to study interference in a task switching situation.

Ninety-six female psychology students took part in these experiments. They were not paid to participate in the study.

There were three independent variables:

- temporal strain was varied by changing the rate of scrolling of the characters on the screen. Two rates were used: low $=18$ characters per minute (one new item every $3.33 \mathrm{~s}$ ), high $=21$ characters per minute (one new item every $2.85 \mathrm{~s}$ ).

- complexity was defined as the amount of information to be processed. Two levels of complexity were used: low $=2$ items, high $=3$ items. Tasks R1, R2 and R5 were considered to be of low complexity, while tasks R3, R4 and R6 were of high complexity.

- similarity was defined by the amount of overlap between the nature of the items and the rules to be applied for execution of the task. The less the overlap the lower the similarity. Thus, tasks R1 and R2 involving selection of even and odd numbers, respectively, were regarded as dissimilar. Likewise there was little similarity between tasks R4 and R6. On the other hand, R1 and R5 were more similar as the first element to be selected is an even number in both cases, and the second element is also a number. For tasks R3 and R6 the only difference is in the second element (letter in R3 
and vowel in R6) so these tasks were also regarded as similar.

A $2 \times 2 \times 2$ design was thus employed with a repeated measure for each sequence. Each group carried out two tasks, $\mathrm{T} 1$ and $\mathrm{T} 2$, of different complexity and similarity. Crossing over with the temporal strain variable defined 8 experimental groups, with a repeated measure for each sequence (Table 1). Twelve participants were assigned to each group at random.

Two dependent variables were analysed: inter-response interval (IRI in sec); and the error rate, expressed as the number of errors divided by the total number of items for each participant.

\subsection{Results}

First, we examined the effects of temporal strain, complexity and similarity on the inter-response interval (IRI) and on the error rate. Secondly, we studied the effects of these variables on the processing when switching from one task to the other. Thirdly, we analysed the temporal evolution of error rate.

\subsubsection{Effects of temporal strain, complexity and similarity on IRI}

A three-way ANOVA with repeated measures showed a significant effect of temporal strain on IRI $\left(F_{1,88}=1377.7, p<0.001\right)$. Under high temporal strain a decrease of IRI for all conditions can be observed. This result can be interpreted as an adjustment to the rhythm of data scrolling.

\subsubsection{Effects of temporal strain, complexity and similarity on error rate}

A three-way ANOVA with repeated measures showed a significant effect of temporal strain on error rate $\left(F_{1,88}=5.741, p<0.02\right)$. Results showed also a near significant effect of complexity $\left(F_{1,88}=3.6, p<0.06\right)$. No interaction effect was observed. High temporal strains and high complexity led to an increase in error rate. Therefore, it seems that the adjustment to the rhythm of data scrolling results in an increasing error rate.

In order to evaluate the effects of the three variables on IRI and error rate in the period following the interruption, we just considered the first $30 \mathrm{~s}$ and last $30 \mathrm{~s}$ of sequences 2, 3, 4, 5 and 6. Data analysis turned on differences between these two periods.

\subsubsection{Effects of temporal strain, complexity and similarity on IRI during task switching}

Three-way ANOVA with repeated measures showed a significant effect of temporal strain on IRI $\left(F_{1}, 88=6.947, p<0.01\right)$. Paradoxically, the difference between the two periods was more pronounced under conditions with low temporal strain than high temporal strain. We assumed that under low temporal strain participants used few processing resources except when they switched from one task to the other.

\subsubsection{Effects of temporal strain, complexity and similarity on error rate during task switching}

Three-way ANOVA with repeated measures showed just an interaction effect between temporal strain and complexity $\left(F_{1}, 88=3.741, p<0.05\right)$. It was in conditions with low temporal strain and high complexity that the difference in error rate between the two periods was the most important. Providing a definite explanation of this paradoxical result would be risky; further experiments, modifying the levels of the temporal strain and complexity, seem necessary in order to propose explanatory hypotheses.

\subsubsection{Temporal course of error rate}

An analysis of the temporal course of mean error rate (without considering each condition separately) showed (Fig. 5) a strong increase of this rate during the first thirty seconds of each sequence. This result supports the hypothesis that the dual process - inhibition and activation of resources - when switching from one task to the other, is costly. On the other hand, the error rate decreases during each sequence and, less clearly, over the repeated sequences.

Table 1

Experimental design ( 8 experimental groups)

\begin{tabular}{llllll}
\hline & Low complexity & & High complexity \\
\cline { 2 - 3 } Low similarity & Low temporal strain & High temporal strain & & Low temporal strain & High temporal strain \\
\hline High similarity & Rules R1R2 & & & Rules R4 R6 \\
\cline { 1 - 2 } & Low temporal strain & High temporal strain & & $\begin{array}{l}\text { Low temporal strain } \\
\text { Rules R3 R6 }\end{array}$ & High temporal strain \\
\hline
\end{tabular}




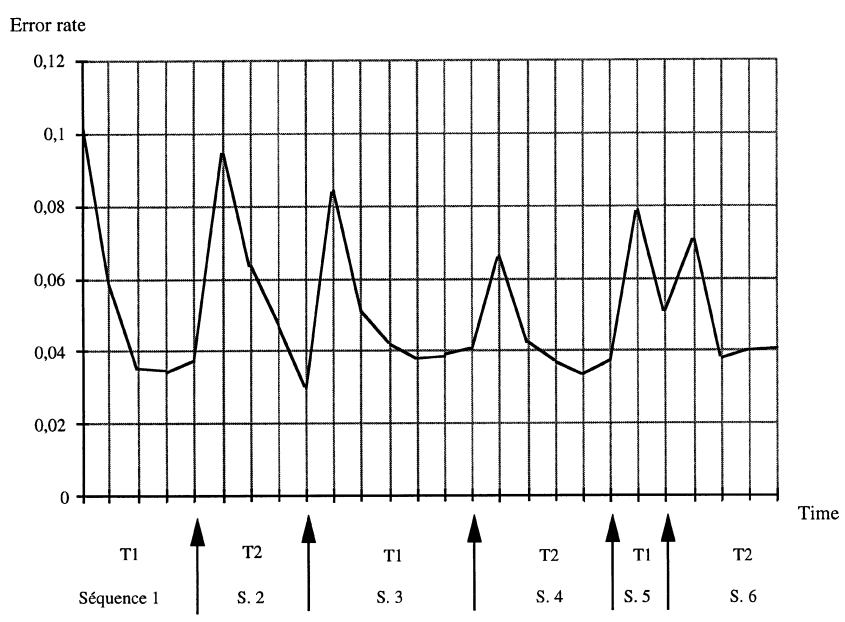

Fig. 5. Temporal course of mean error rate (the first minute of the sequences 2,3,4,5 and 6 is divided into two periods of $30 \mathrm{~s}$, the end of these sequences is graduated in minutes).

A qualitative analysis of errors has been made (Cellier and Eyrolle, 1992). We distinguished two types of error:

- time-sharing non-specific errors (errors of manipulation and anticipation observed in both single task and switched tasks);

- time-sharing specific errors (observed mainly in switched tasks) divided into three subgroups: intrusions (the participant makes a correct response to task 1 while carrying out task 2); omissions (the participant misses items obeying the relevant rule); and confusions (the participant selects two items, each one obeying a part of each rule). For example, the participant has just switched from R1 (select even numbers followed by an odd number) to R2 (select odd numbers followed by an even number) but he selects the items 35/43. The first item selected corresponds to $\mathrm{R} 2$ but the second one corresponds to R1. Specific errors appeared more sensitive to interruptions than the nonspecific ones.

\section{Discussion}

Both studies presented in this paper were carried out in order to evaluate the effects of interruptions in work activity. We are not going to compare the results between the studies since the tasks involved and the conditions under which data were collected were not similar. The field study can be viewed as an exploratory study and it allowed us especially to verify the relevance of the problem of task interruption.

The results obtained in the field study showed, on the one hand, an increase of the processing time of the first task when it was shared, due to interruptions. This result suggests the existence of some proximal after-effects (Roe et al., 1996) and not only a momentary constraint due to the interruption. Results did not show, on the other hand, any significant increase in the error rate. We supposed that the constraints induced by the interruptions were compensated in this situation by an increase of the processing time that allowed minimisation of the errors. Indeed, in this situation there were not high temporal strains. One curious finding lies in the frequency of use of the strategies identified. Both strategies (strategies 1 and 2) that are to complete the processing of the current task before the processing of the interruptive task are little used. Nevertheless, they seem apt to limit the impact of the interruptions on the execution of the main task. We hypothesised that the choice of this or that strategy by the operator could be influenced by two classes of determinants, external and internal. The priority generally conceded to the processing of the interruptive task can be accounted for by the commercial policy of the organisation that recommended immediate answers to customers' calls. Meanwhile, if this external determinant accounts for most of the cases (in $77 \%$ of the cases, strategy 4 is used), it cannot explain the choice for the three other strategies. Then we supposed that internal determinants became influential; when the interruptive task occurs the operator evaluates different parameters, such as the time needed to complete the current task or the consequences of the interruption on the processing of the current task, in terms of mental effort or error risk. This explanation is coherent with the results of Gillie and Broadbent (1989), who noted that the nature of the interruption and its complexity would determine the disruptive (or not disruptive) character of the interruption.

These results have then oriented the research towards the role of several factors on time-sharing efficiency. Temporal strains, complexity and similarity of tasks have been manipulated in an experimental design. Results concerning inter-response intervals are not convincing. We observed a significant effect of temporal strains on the whole task (high temporal strains led to decreased intervals) that we interpreted as an adjustment to the rhythm of data scrolling, and a significant effect of temporal strains during task switching (under low temporal strains, differences between the first thirty and last thirty seconds were greater than under high temporal strains). Results about error rates are more interesting as they showed a significant effect of temporal strains (high temporal strains led to an increased error rate) and some effect of complexity (high complexity led to an increased error rate). These results support the hypothesis of a greater difficulty under higher demands to bring the management resource strategy into play. The strong increase of the error rate in the first thirty seconds of processing of each sequence seems to support the hypothesis of the dual process of activation and inhibition in resource allocation, although we could not assess their 
degree of interdependency. Finally, we did not observe any effect of similarity on inter-response intervals or on error rates. Maybe the way this variable had been defined does not allow a consideration of two contrasted levels of similarity. In fact, tasks were very similar in all conditions. Therefore, the effect of this factor is surely important especially for the accuracy of the responses. Finally, further investigations would be necessary in order to study thoroughly the determinants of choice for different strategies when an interruption occurs, in order to propose methods which limit errors and also preserve operators' psychological well-being.

\section{Conclusion}

From an operational point of view, system designers and people in charge of work organisation have to take into account human sensitivity to task interruptions, where the most frequent consequences are lower performance, errors and stress. They have to propose technical and organisational systems to reduce the incidence or consequences of interruptions. Two main complementary ways are possible. The first way is use of technological tools; the classical option is to allow an immediate saving of the current file and to offer a rapid and easy return to the field being worked on. The power of present computers permits several technical solutions in order to satisfy this requirement.

The second way is through work organisation. The person in charge of work organisation should know the potential consequences of task interruptions in order to evaluate their costs. The organisational response will then be a redistribution of tasks between the operators, allowing reduction in the probability of interruptions. This response may be completed by technological solutions previously underlined. Both options have in common increasing the controllability of the work environment, defined by Hockey, Briner, Tatersall and Wiethoff (1989) as "the extent to which it provides the operator with the opportunity for exercising discretion in the planning and execution of work activities" (p. 1403).

\section{Acknowledgements}

The laboratory study was funded by the DRET/Ministry of Defence (contract number 86.34.109.00.470.75.01).

\section{References}

Bainbridge, L., 1984. Diagnostic skills in process operation. In: Proceedings of 1984. International Conference on Occupational Ergonomics, Toronto, Vol. 2, pp. 1-10.

Broadbent, D.E., 1958. Perception and communication. Pergamon Press, New York.

Cellier, J.-M., Eyrolle, H., 1992. Interference between switched tasks. Ergonomics 35, 25-36.

Carayon, P., 1994. Stressful jobs and non-stressful jobs: a cluster analysis of office jobs. Ergonomics 37, 311-323.

Gillie, T., Broadbent, D., 1989. What makes interruptions disruptive? A study of length, similarity and complexity. Psychol. Res. 50, 243-250.

Griffon-Fouco, M., Ghertman, F., 1984. Recueil de données sur les facteurs humains à Electricité de France. In: Operational Safety of Nuclear Power Plants. International Atomic Energy Agency, Vienna, pp. 157-172.

Hockey, G.R.J., Hamilton, P., 1983. The Cognitive Patterning of Stress States. In: Hockey, G.R.J. (Ed.), Stress and Fatigue in Human Performance. Wiley, New York, pp. 331-361.

Hockey, G.R.J., Briner, R.B., Tatersall, A.J., Wiethoff, M., 1989. Assessing the impact of computer workload on operator stress: the role of system controllability. Ergonomics 32 (11), 1401-1418.

Kahneman, D., 1973. Attention and Effort. Prentice-Hall, Englewood Cliffs, NJ.

Navon, D.A., Gopher, D., 1979. On the economy of the human processing system. Psychol. Rev. 86, 214-255.

Norman, D.A., Bobrow, D.G., 1975. On data-limited and resource limited processes. Cognitive Psychol. 7, 44-64.

Posner, M.I., Snyder, C.R.R., 1975. Facilitation and inhibition in the processing of signals. In: Rabitt, P.M.A., Dornic, S. (Eds.), Attention and Performance. Academic Press, New York, pp. 669-682.

Reason, J., 1990. Human Error. Cambridge University Press, Cambridge.

Roe, R.A., Zijlstra, F.R.H., Leonova, A.B. 1996. Interruptions in mental information work. Effects on work activitiy and psychological wellbeing. WORC Report 96.06.003/4, Tilburg University Press, Tilburg.

Shiffrin, R.M., Schneider, W., 1977. Controlled and automatic human information processing: 2 - Perceptual learning, automatic attending and a geneal theory. Psychol. Rev. 84, 127-190.

Treisman, A.M., 1964. Selective attention in man. Br. Med. Bull. 20, 12-16.

Welford, A.T., 1967. Single channel operation in the brain. Acta Psychol 27, 5-22.

Wickens, C.D., 1984. Processing resources in attention. In: Parasuraman, R., Davies, P.R. (Eds.), Varieties of Attention. Academic Press, Orlando, pp. 63-102. 